\title{
NOTIZEN
}

\section{Production of a Dense Thermonuclear Plasma by a Pulsed High Intensity Field Emission Discharge*}

F. WinterberG **

International Centre for Theoretical Physics, Trieste, Italy

(Z. Naturforsch. 23 a, 1396—1397 [1968] ; received 23 July 1968)

A new method to controlled fusion is proposed which is based on a recently developed technique ${ }^{1-4}$ to produce very intense and energetic electron beams for a period on the order of a few nanoseconds. In one of these reported experiments ${ }^{1}$, the electron energy was approximately $10 \mathrm{MeV}$, the total beam current $3 \times 10^{5}$ amp, and the discharge time 10 nanoseconds. From these data, it follows that the total energy output was $3 \times 10^{4}$ joule $=3 \times 10^{11} \mathrm{erg}$. In another experiment ${ }^{3}$, the electron beam struck a solid target creating extremely strong shock waves.
The energy for the pulsed field emission discharge is drawn from a large capacitor bank by means of the Marx circuit technique. The electrons are extracted from a concave brush of field emitter cathodes. If their space charge is neutralized by a plasma, then the electrons can be collimated into a narrow beam because of their high self-magnetig field. The neutralizing plasma can be generated by the evaporation of the target surface. For the same purpose one can place a tenuous plasma in front of the target.

By the further development of this method, it should be possible to arrive at critical conditions under which the ignition of a small thermonuclear explosion can be achieved, if the target is made out of a sufficiently large amount of liquid or solid T-D, preferably surrounded by a heavy substance to increase the inertial confinement after ignition. The technical limitations posed against the possibility to achieve the same goal by a giant pulsed laser do not exist in our case. The principle of the idea is indicated in Fig. 1 .

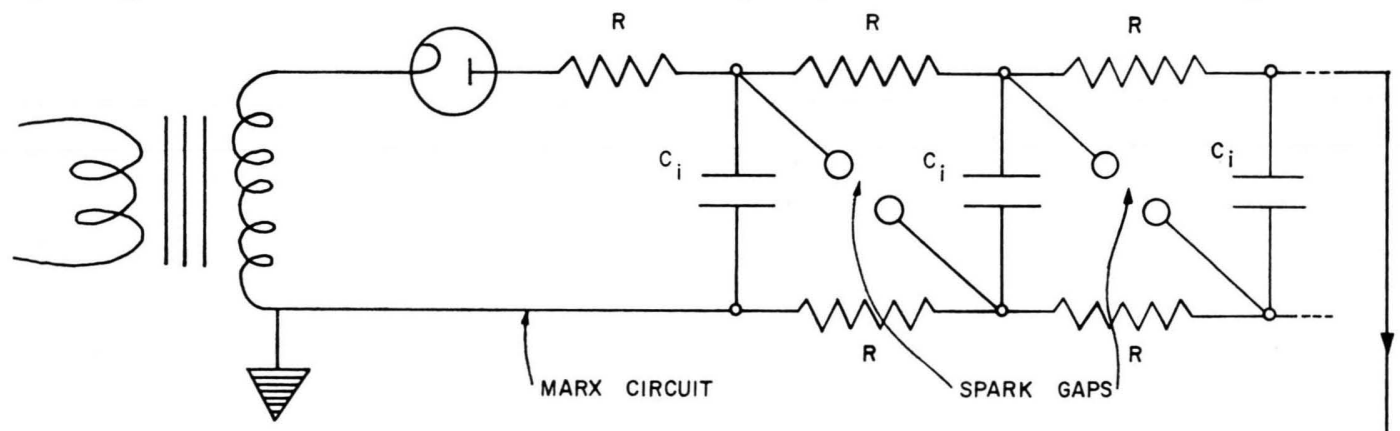

1) The target has to be heated above the ignition temperature for the T-D thermonuclear reaction

$$
T>5 \times 10^{7}{ }^{\circ} \mathrm{K} .
$$

2) In order to obtain a detonation, the fusion products have to remain within the target.

The range of fusion products in T-D at liquid densities and thermonuclear temperatures is approximately $0.4 \mathrm{~cm}$, requiring a target radius $r$ of solid or liquid T.D, $r>0.2 \mathrm{~cm}$.

3) The Lawson criterion $N \tau$ $\geqq 10^{14} \mathrm{sec} / \mathrm{cm}^{3}$ must be satisfied. For an unconfined plasma the
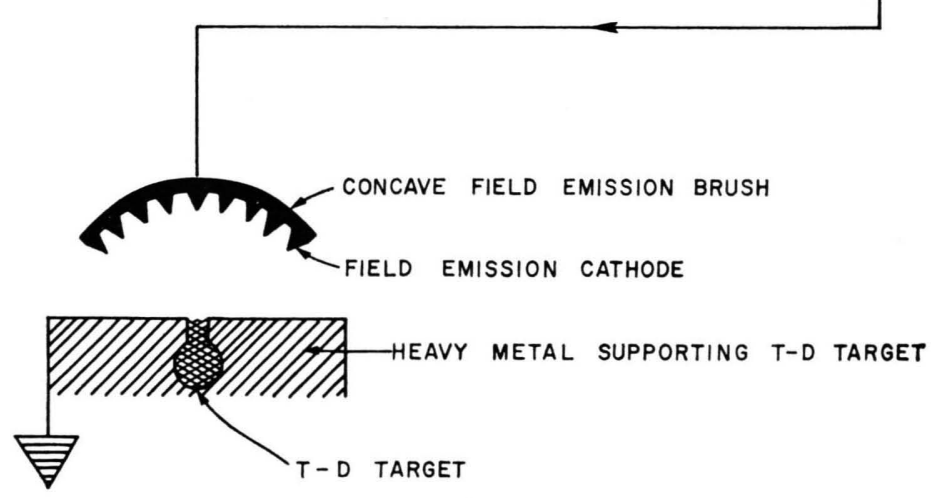

FIG. I

* Supported in part by the National Aeronautics and Space Administration under Grant No. NGR-29-001-016.

** On leave from the Desert Research Institute and Department of Physics, University of Nevada, Reno, Nevada, USA. 
largest energy loss results from expansion. The expansion time for a T-D sphere of $0.25 \mathrm{~cm}$ radius is given by $2.5 \times 10^{-9}$ sec. The next important loss results from bremsstrahlung with a characteristic time of $5 \times 10^{-8}$ sec. The expansion losses can be reduced by surrounding the target with some heavy high $A$ number material. In this case losses by electronic heat conduction lead to a characteristic time for the given example of $2.6 \times 10^{-8} \mathrm{sec}$ at $5 \times 10^{7} \mathrm{~K}$ and $4 \times 10^{-9} \mathrm{sec}$ at $10^{8}{ }^{\circ} \mathrm{K}$. It follows that, bz surrounding the target with a heavy substance, the Lawson criterion can be satisfied.

4) The energy required for ignition must be supplied to the target in a time shorter than $5 \times 10^{-8} \mathrm{sec}$. Assuming that $r=0.25 \mathrm{~cm}$, one obtains the total energy to be absorbed in the T-D target

$$
\varepsilon=4 \pi N k T r^{3}=5.4 \times 10^{13} \mathrm{erg}=5.4 \text { megajoule. }
$$

It follows that this is about $10^{2}$ to $10^{3}$ larger than the energy output in the devices quoted above.

The field emission current density $j$ at each emitter cathode is determined by

$j=1.55 \times 10^{-6}\left(E^{2} / W\right)$

$$
\cdot \exp \left[-6.9 \times 10^{7} W^{3 / 2} / E\right]\left(\mathrm{amp} / \mathrm{cm}^{2}\right),
$$

where $E$ is the electric field strength in volt/cm and $W$ is the work function in $\mathrm{eV}$. The electric field is expressed by the applied voltage through $E=V / r$, where $r$ is the radius of the field emitter tip. If we assume $V=10^{7}$ volt, $r=0.1 \mathrm{~cm}$, then one finds easily that the total current emitted from one cathode with the area $2 \pi r^{2}$ is given (for a tungsten cathode) by $I=3.5 \times 10^{5}$ amp, and the power output is $P=I V=3.5 \times 10^{12}$ watt.

1 F. C. Ford, D. Martin, D. Sloan, and W. Link, Bull. Am. Phys. Soc. 12, 961 [1967].

2 S. E. Graybill and S. V. Nablo, Bull. Am. Phys. Soc. 12, 961 [1967].

3 F. J. Grundhauser, J. L. Brester, J. B. Barbour, F. M. Charвonnier, and W. P. Dyke, Bull. Am. Phys. Soc. 12, 961 [1967].
The delivery of $10^{14} \mathrm{erg}$ in 10 nanoseconds would hence require a brush of approximately 300 field emission cathodes.

It can be shown that the electron beam can deposit its energy by the mechanism of the counterstream instability. For the maximum current density which is $10^{10} \mathrm{amp} / \mathrm{cm}^{2}$, reached in the focus, the length over which the beam will be dissipated can be calculated from the maximum growth rate for the counterstream instability ${ }^{4}$. In dense T-D it is of the order of $10^{-4} \mathrm{~cm}$ and hence sufficiently short.

In case the mechanism of beam dissipation by the counterstream instability should be unfeasible for reasons not yet anticipated one may try to use a field ion emisison ${ }^{5}$. The range of $\mathrm{MeV}$ protons in T-D at liquid densities and thermonuclear temperatures is of the order $\mathrm{cm}$, and therefore short enough to ensure large energy absorption in the T-D target even without dissipation by the counterstream instability. However, in contrast to field electron emission, the ions for the field ion emission have to be supplpied from an outside source.

To produce a high field ion current, it is proposed to use emitter anodes with a central hole through which un-ionized deuterium gas can flow to the emitter tip. The gas is forced by high pressure into the hole. If the electric field at the emitter tip is larger than $10^{8}$ volt $/ \mathrm{cm}$, then the gas atoms will be stripped of their electrons by field ionization resulting in an intense field ion emission current. It can be easily shown that by this method a field ion current of sufficient intensity can be produced.

4 O. Buneman, Phys. Rev. 115, 503 [1959].

5 F. Winterberg, International Centre for Theoretical Physics, Trieste, Internal Report 35/1967 to be published in Phys. Rev. 